\title{
Treatment Strategy for Impending Instability in Spinal Metastases
}

\author{
Yeon Ho Kim, MD, Junho Kim, MD, Sam Yeol Chang, MD, Hyoungmin Kim, MD, Bong-Soon Chang, MD \\ Department of Orthopedic Surgery, Seoul National University Hospital, Seoul National University College of Medicine, Seoul, Korea
}

\begin{abstract}
Background: Determining surgical management of a spinal metastasis is difficult owing to the involvement of multiple factors. The spinal instability neoplastic score (SINS) system is a reliable tool to evaluate instability in spinal metastases. The intermediate SINS (scores 7-12) indicates impending instability, which makes it difficult to determine the proper treatment strategy. In this study, we aimed to compare the initial status and treatment outcomes of a conservative group versus an operative group among patients with spinal metastases with an intermediate SINS of 7-12. Further, we evaluated the time for conversion to surgery in patients who had initially undergone conservative treatment and identified the factors associated with the conversion.

Methods: Among the patients with a spinal metastasis with an intermediate SINS of 7-12 from May 2013 to December 2017, those who were followed up for more than 12 months were enrolled in this study. Patients with signs of a neurologic deficit or cord compression at the initial diagnosis were excluded. Finally, 79 patients ( 47 in the initially conservative group and 32 in the initially operative group) were enrolled in this study. The performance status, Tomita score, and Tokuhashi score were assessed for group comparison. Components of SINS, the Bilsky grade, and radiosensitivity of tumor were evaluated to determine factors associated with conversion to surgery.

Results: Average follow-up was 20.9 months (range, 12-46 months). The demographic variables, primary cancer type, and performance status were not significantly different between the 2 groups. However, the Tomita score was lower in the initially operative group $(p=0.006)$. The 1 -year treatment outcome assessed based on the change in performance status and vertebral height collapse showed a tendency to deteriorate less in the initially operative group. The rate of conversion to surgery in the initially conservative group was $33 \%$ in the first year, after which there was little change in the incidence of conversion. When vertebral body collapse was less than $50 \%$ or the tumor was located in the semi-rigid region (T3-T10), the need for conversion to surgery increased statistically significantly ( $p=0.039$ and $p=0.042$, respectively).

Conclusions: The rate of conversion to surgery in initially conservatively treated patients was about $33 \%$ in the first year. When a tumor is located in T3-T10 and less than $50 \%$ vertebral body collapse is present, surgery may be the better choice than conservative treatment.
\end{abstract}

Keywords: Spinal metastasis, Spinal instability neoplastic score, Impending instability, Survival curve

The incidence rate of cancer is expected to increase, resulting in an increase in spinal metastases. Decision-making

Received January 21, 2020; Accepted February 18, 2020

Correspondence to: Bong-Soon Chang, MD

Department of Orthopedic Surgery, Seoul National University Hospital, 101

Daehak-ro, Jongno-gu, Seoul 03080, Korea

Tel: +82-2-2072-3864, Fax: +82-2-764-2718

E-mail: bschang@snu.ac.kr for surgical management of spinal metastasis is challenging because there are many factors to consider. Treatment decision tools such as the LMNOP system and NOMS framework have emerged in the last few years. ${ }^{1,2}$ These systems allow for excellent qualitative assessment of spinal metastases but cannot be used for quantitative evaluation. The Spinal Oncology Study Group introduced the spinal instability neoplastic score (SINS) system. ${ }^{3)}$ The SINS system is considered as a reliable tool to determine instability 
Kim et al. Impending Instability in Spinal Metastasis

Clinics in Orthopedic Surgery • Vol. 12, No. 3, $2020 \bullet$ www.ecios.org

in spinal metastases. It is based on radiological findings and clinical pain and has 3 categories: $0-6$ points, stability; 7-12, impending instability; and 13-18, instability (Table 1). Among these categories, the intermediate SINS (7-12 points), which indicates impending instability, causes difficulty in deciding whether and when to operate.

In this study, we aimed to evaluate the rate and time of conversion to surgery in patients with an intermediate SINS who had undergone conservative treatment and to identify the factors associated with conversion to surgery.

Table 1. Components of Spinal Instability Neoplastic Score

\begin{tabular}{lc} 
Components of spinal instability neoplastic score & Score \\
Location & \\
\hline Junctional (Occiput-C2, C7-T2, T11-L1, L5-S1) & 3 \\
\hline Mobile spine (C3-6, L2-4) & 2 \\
Semi-rigid (T3-T10) & 1 \\
\hline Rigid (S2-5) & 0 \\
Pain & \\
\hline Yes & 3 \\
Occasional pain but not mechanical & 2 \\
Pain-free lesion & 0
\end{tabular}

Bone lesion

Lytic 2

Mixed (Iytic/blastic) 1

Blastic

0

Spinal alignment

Subluxation/translation present

4

De novo deformity (kyphosis/scoliosis)

2

Normal alignment

0

Vertebral body collapse

$>50 \%$ collapse

3

$<50 \%$ collapse

2

No collapse with $>50 \%$ body involved

None of the above

1

Posterolateral involvement of the spinal elements

Bilateral

3

Unilateral

1

None of the above

\section{METHODS}

A consecutive series of patients with spinal metastases and an intermediate SINS (7-12 points) from May 2013 to December 2017 were retrieved from the electronic medical record system of the Orthopedic Clinic of Seoul National University Hospital. Patients who had been followed up for more than 12 months were enrolled in this study. Patients who had initially neurologic deficits or signs of cord compression were excluded because these patients were indicated for surgery. The study was approved by the Institutional Review Board of Seoul National University hospital (IRB No. H-1911-018-1076). As this study was based on a retrospective review of the past medical records, the need for informed consent was waived.

The decision to perform surgery was made in a weekly tumor board meeting consisting of various specialists involved in the treatment of spinal metastases. Multiple factors including primary cancer type, performance status, prognosis, systemic therapy sensitivity, radiotherapy sensitivity, degree of pain, and neurologic deficit were considered in the final decision. Patients were divided into 2 groups (initially conservative group and initially operative group), and demographic data, pretreatment baseline factors, and treatment outcomes were compared between the 2 groups.

We collected demographic data of the patients, including sex, age, and primary cancer. The Tomita scoring system, revised Tokuhashi scoring system, and SINS system were used, and the Karnofsky performance status score and Eastern Cooperative Oncology Group (ECOG) score were used for the assessment of the initial status and treatment outcome.

For evaluation of the rate of conversion to surgery during the follow-up period in the group that initially underwent conservative treatment, we used survival analysis. To determine the associated factors in conversion to surgery in the initially conservative group, the total score and each component of the SINS system, degree of epidural canal compromise by the Bilsky grade, sensitivity of radiotherapy, ${ }^{4,5)}$ and initial performance status were analyzed.

For statistical evaluation, Student $t$-test and chisquare test were applied for comparison between groups. Survival analysis was applied to assess the rate of conversion to surgery in the conservative group and determine the associated factors. The log-rank test was used for survival analysis. A $p$-value $<0.05$ was considered statistically significant. For statistical analysis, IBM SPSS ver. 23.0 (IBM Corp., Armonk, NY, USA) was used. 
Kim et al. Impending Instability in Spinal Metastasis

Clinics in Orthopedic Surgery • Vol. 12, No. 3, $2020 \bullet$ www.ecios.org

\section{RESULTS}

From May 2013 to December 2017, 79 patients with the intermediate SINS (initially conservative group, $\mathrm{n}=47$; initially operative group, $\mathrm{n}=32$ ) were analyzed in this study. Their mean age was 61.2 years (range, $36-82$ years), and the mean follow-up period was 20.9 months (range, 12-60 months) (Fig. 1). Table 2 summarizes the demographic information and primary cancer type of the patients. The common tumors that metastasized to the spine were lung $>$ breast and colon > liver, kidney, and thyroid.

The difference in the initial performance status (based on Karnofsky and ECOG scores) between the initially conservative and initially operative groups was not statistically significant ( $p=0.953$ and $p=0.683$, respectively). Further, the SINS and initial vertebral height loss were not significantly different $(p=0.875$ and $p=0.385$, respec-

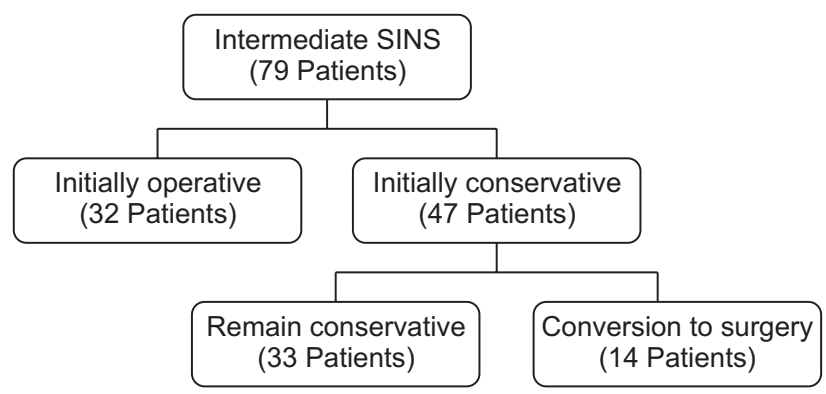

Fig. 1. Patients enrollment. SINS: spinal instability neoplastic score.

Table 2. Demographic Information and Types of Primary Cancer

\begin{tabular}{|cccc|}
\hline \multicolumn{1}{c}{ Variable } & $\begin{array}{c}\text { Initially } \\
\text { conservative }\end{array}$ & $\begin{array}{c}\text { Initially } \\
\text { operative }\end{array}$ & $\begin{array}{c}p \text {-value or } \\
\text { ratio }(\%)\end{array}$ \\
\hline Age (yr) & $58.3 \pm 12.7$ & $63.2 \pm 11.4$ & 0.228 \\
\hline Sex (male : female) & $29: 18$ & $17: 15$ & 0.447 \\
\hline Primary cancer & 9 & 2 & $11(18)$ \\
\hline Lung & 6 & 3 & $9(11)$ \\
\hline Breast & 5 & 3 & $8(10)$ \\
\hline Liver & 5 & 4 & $9(11)$ \\
\hline Colon & 4 & 1 & $5(6)$ \\
\hline Prostate & 4 & 4 & $8(10)$ \\
\hline Kidney & 3 & 0 & $3(4)$ \\
\hline Multiple myeloma & 2 & 6 & $8(10)$ \\
\hline Thyroid & 9 & 6 & $15(19)$ \\
\hline Other & & & \\
\hline
\end{tabular}

tively) between the groups; however, the initially operative group had significantly lower Tomita scores $(p=0.006)$ and higher revised Tokuhashi scores $(p=0.119)$, which suggests that surgery resulted in a better prognosis. The 1 -year treatment outcome was evaluated by the deterioration of the performance status and vertebral collapse ratio. The initially operative group had a tendency to show less deterioration in the Karnofsky score and vertebral body collapse ( $p=0.064$ and $p=0.061$, respectively)

In the initially conservative group $(n=47), 14$ patients $(25.5 \%)$ were converted to surgery during the follow-up period. The rate of conversion to surgery using survival analysis was approximately $33 \%$ in the first year (Fig. 2). However, less conversion was required in the subsequent years. The rate of conversion to surgery was not statistically significantly different among the patients divided into 2 and 3 groups according to their SINS ( $p=$ 0.646 and $p=0.945$, respectively). Each component of the SINS system (mechanical pain, bone lesion, radiographic spinal alignment, and posterolateral involvement) had no statistically significant impact on the rate of conversion to surgery. The degree of epidural canal compromise and the degree of radiosensitivity according to the type of tumor were not significantly associated with the risk of conversion to surgery ( $p=0.189$ and $p=0.345$, respectively). However, less than 50\% vertebral body collapse and the semi-rigid location (T3-T10) of a tumor were statistically significant risk factors for conversion to surgery ( $p=0.039$ and $p=0.042$, respectively) (Fig. 3).

\section{DISCUSSION}

Assessing stability in patients with spinal metastases is im-

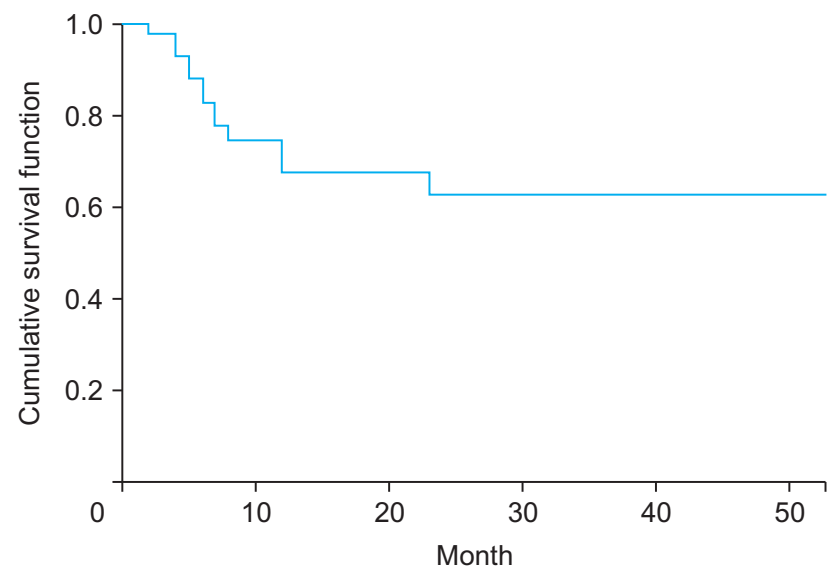

Fig. 2. Survival curve of conversion rate to surgery in conservative group. In the first year, 33\% of the conservatively treated patients underwent surgery. 
Kim et al. Impending Instability in Spinal Metastasis

Clinics in Orthopedic Surgery • Vol. 12, No. 3, 2020 • www.ecios.org

A

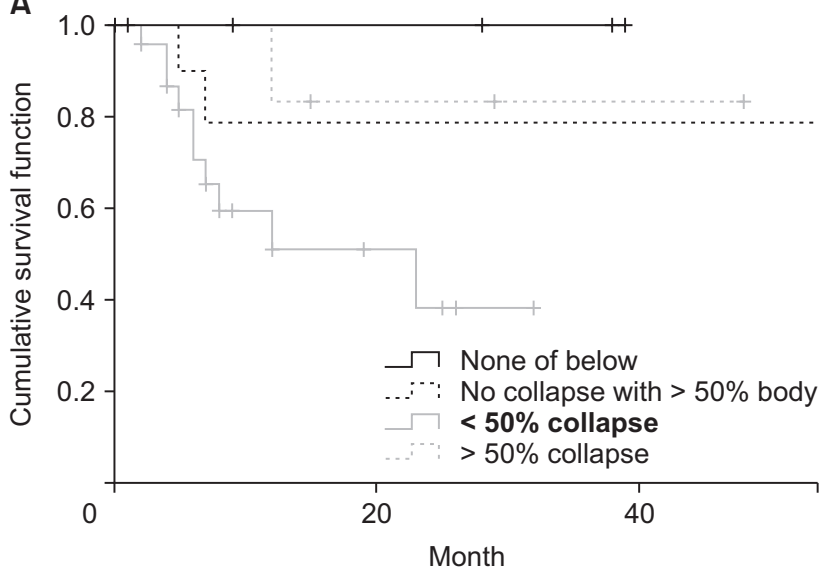

B

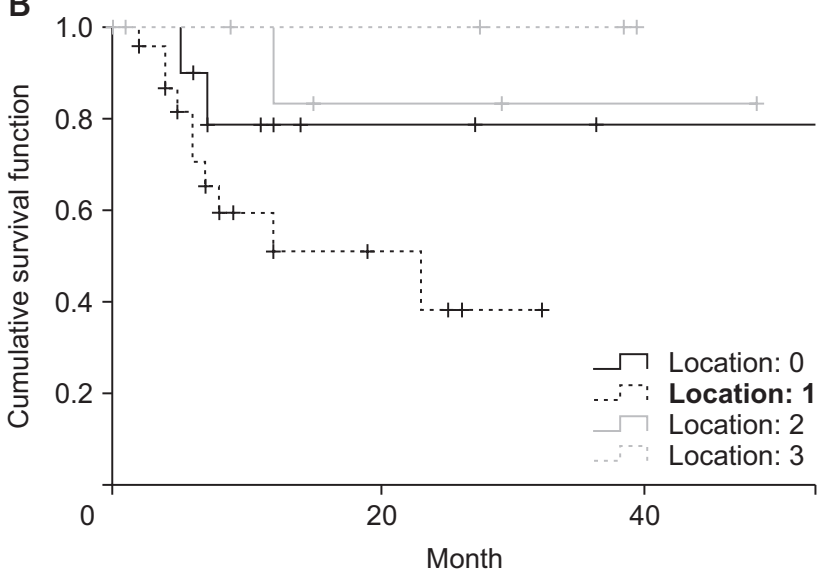

Fig. 3. Survival analysis according to degree of vertebral body collapse $(A)$ and tumor location (B). Vertebral body collapse less than $50 \%$ or metastasis located in semi-rigid region (T3-T10) showed a statistically significant conversion rate to surgery.

portant to determine the proper treatment strategy. There have been many efforts to evaluate and classify the stability of the spine with metastatic lesions. Harrington classified spinal metastases into 5 categories on the basis of bone and neurologic involvement and suggested treatment options for each category. ${ }^{6}$ Kostuik proposed a 6 column system to develop spinal instability criteria, ${ }^{7)}$ and Taneichi et al. ${ }^{8)}$ investigated the risk factors for and probability of vertebral body collapse in the thoracolumbar spine with metastatic lesions. Besides, the risk factors for intervertebral instability, including axial rigidity, ${ }^{9)}$ location of metastasis within the vertebral body, ${ }^{2)}$ tumor size, ${ }^{10)}$ and decreased bone density, ${ }^{10,11)}$ have been reported. However, these classification systems and risk factors have not been validated, and the systematic review of Weber et al. ${ }^{12)}$ has not provided a satisfactory conclusion.

The SINS system has been widely used to evaluate the structural stability of the spine in patients with metastases. It is a comprehensive, easy-to-use system that assesses intervertebral instability based on 5 imaging data and 1 clinical component score: spinal level of the lesion, presence and type of pain, bone quality of the lesion, spinal alignment, extent of vertebral body collapse, and posterolateral involvement of the spinal elements. SINS ranging from 0 to 18 points are classified as follows: $0-6$ points, stability; 7-12 points, impending instability; and 13-18, instability. Many studies supported the high reliability of the SINS system. ${ }^{13-16)}$ However, it is difficult to determine whether a surgical intervention is indicated for the intermediate category (scores 7-12, impending instability), which has been discussed in only a few studies. ${ }^{17,18)}$

In this retrospective study, the rate of conversion to surgery among patients with an intermediate SINS who
Table 3. Causes for Conversion to Surgery in the Initially Conservative Group

\begin{tabular}{rrrrl} 
Case & $\begin{array}{r}\text { Initial } \\
\text { SINS }\end{array}$ & $\begin{array}{r}\text { Preop } \\
\text { SINS }\end{array}$ & Lesion & Reason for surgery \\
\hline 1 & 9 & 9 & L2 & Neurologic Sx \\
\hline 2 & 8 & 9 & L4 & Neurologic Sx \\
\hline 3 & 12 & 13 & T4 & Neurologic Sx \\
\hline 4 & 8 & 8 & T4 & Neurologic Sx \\
\hline 5 & 11 & 11 & T10 & Neurologic Sx \\
\hline 6 & 8 & 10 & L4 & Neurologic Sx \\
\hline 7 & 9 & 9 & T2 & Neurologic Sx \\
\hline 8 & 10 & 11 & T3 & Neurologic Sx \\
\hline 9 & 11 & 11 & C2 & Neurologic Sx \\
\hline 10 & 11 & 11 & T6 & Neurologic Sx \\
\hline 11 & 12 & 12 & T10 & Severe back pain \\
\hline 12 & 9 & 10 & C7 & Severe back pain \\
\hline 13 & 8 & 8 & L1 & Severe back pain \\
\hline 14 & 11 & 13 & T11 & Severe collapse \& pain \\
\hline S & 12 & & & \\
\hline
\end{tabular}

SINS: spinal instability neoplastic score, Preop: preoperative, Sx: symptom.

had initially undergone conservative treatment was approximately $33 \%$ in the first year, with little change in the following period. These results show that approximately one-third of the cases experience progression to surgery within 1 year after conservative treatment, and if instability does not get worse within the first year, it does not 
Kim et al. Impending Instability in Spinal Metastasis

Clinics in Orthopedic Surgery • Vol. 12, No. 3, $2020 \bullet$ www.ecios.org

progress thereafter.

When identifying associated factors in conversion to surgery, our first hypothesis was that the higher the SINS, the more likely the patients were to undergo surgery. However, no significant difference was observed among patients divided into 2 groups and 3 groups according to the SINS. Besides, the components of SINS, including the presence and type of pain, bone quality of the lesion, spinal alignment, and posterolateral involvement of the spinal elements, showed no significant association with the conversion to surgery. However, there was a statistical significance in the risk of conversion to surgery when a tumor was located in the semi-rigid thoracic region (T3T10) or there was less than $50 \%$ vertebral body collapse. This result seems to be not in line with to the SINS system, which considers tumors in semi-rigid regions as favorable. In the opinion of the authors, this may be attributed to the reason for conversion to surgery. Among the 14 patients who underwent surgery later, the reason for surgery in 12 patients was neurologic deficits owing to cord compression (Table 3). ${ }^{19)}$

To determine the association with the initial canal status, the Bilsky grade was used as a variable; however, it showed no statistically significant difference between the groups. This result could be attributed to the relatively narrow spinal canal at the thoracic vertebra and collapsed vertebral body in the thoracic region, which could have facilitated cord compression in these areas. Further, patients with thoracic spine tumors and an intermediate SINS had higher scores for other components, which could have caused higher instability. Patients with less than 50\% vertebral collapse had a higher risk to require surgery mainly due to further vertebral collapse. More than 50\% collapse of the vertebral body at the initial diagnosis seemed to have less chance of worsening in patients with an intermediate SINS

Radiosensitivity of the tumor was analyzed to see if conversion to surgery was related to the tumor type. Tumors were divided into 2 categories: radiosensitive tumors (i.e., thyroid, breast, colon, and non-small cell lung cancer) and radioresistant tumors (i.e., melanoma, renal cell carcinoma, and sarcoma). ${ }^{5}$ There was no statistically significant difference between 2 groups. However, it was difficult to determine relationships because the tumor types were diverse and radiation protocols varied.

Between the initially operative and initially conservative groups, we compared the initial performance status (Karnofsky and ECOG scores), initial vertebral height collapse ratio, Tomita score, revised Tokuhashi score, and SINS for the evaluation of demographic data and pretreatment baseline factors. The Tomita and revised Tokuhashi scores are commonly used to determine the prognosis of spinal metastases. ${ }^{20-23)}$ No significant differences were observed in the initial performance status and initial vertebral height collapse ratio. However, the Tomita scores were statistically significantly lower $(p=0.006)$ and the revised Tokuhashi scores were higher $(p=0.119)$ in the initially operative group, indicating that surgery over conservative treatment was done in patients whose prognosis was expected to be better. There seemed to be an inevitable difference in deciding a treatment. ${ }^{24)}$

There are some limitations in this study. First, the cohort of this study was heterogeneous, and analysis was difficult as the patients had different tumor types and prognoses, and thus in the follow-up period, the patients were managed by various treatment plans. Second, this study included a small sample size owing to the narrow inclusion criteria; hence, the statistical power of the study was low. Third, selection bias might have been introduced owing to the retrospective nature of the study. Despite these limitations, this might be the first study to analyze the prognosis and the associated factors leading to conversion to surgery in patients with impending instability who had been initially treated conservatively.

In conclusion, it is difficult to decide whether or not to operate spinal metastases with an intermediate SINS (scores 7-12), which indicate impending instability. In patients who initially underwent conservative treatment, the rate of conversion to surgery was approximately $33 \%$ in the first year and there was little change in the following period. Surgery is recommended over conservative treatment for tumors present in T3-T10 or with less than 50\% initial vertebral body collapse.

\section{CONFLICT OF INTEREST}

No potential conflict of interest relevant to this article was reported.

\section{REFERENCES}

1. Paton GR, Frangou E, Fourney DR. Contemporary treatment strategy for spinal metastasis: the "LMNOP" system.
Can J Neurol Sci. 2011;38(3):396-403.

2. Laufer I, Rubin DG, Lis E, et al. The NOMS framework: 
Kim et al. Impending Instability in Spinal Metastasis

Clinics in Orthopedic Surgery • Vol. 12, No. 3, $2020 \bullet$ www.ecios.org

approach to the treatment of spinal metastatic tumors. Oncologist. 2013;18(6):744-51.

3. Fisher CG, DiPaola CP, Ryken TC, et al. A novel classification system for spinal instability in neoplastic disease: an evidence-based approach and expert consensus from the Spine Oncology Study Group. Spine (Phila Pa 1976). 2010; 35(22):E1221-9.

4. Katsoulakis E, Kumar K, Laufer I, Yamada Y. Stereotactic body radiotherapy in the treatment of spinal metastases. Semin Radiat Oncol. 2017;27(3):209-17.

5. Bernard V, Bishop AJ, Allen PK, et al. Heterogeneity in treatment response of spine metastases to spine stereotactic radiosurgery within "Radiosensitive" subtypes. Int J Radiat Oncol Biol Phys. 2017;99(5):1207-15.

6. Harrington KD. Metastatic disease of the spine. J Bone Joint Surg Am. 1986;68(7):1110-5.

7. Kostuik JP, Errico TJ, Gleason TF, Errico CC. Spinal stabilization of vertebral column tumors. Spine (Phila Pa 1976). 1988;13(3):250-6.

8. Taneichi H, Kaneda K, Takeda N, Abumi K, Satoh S. Risk factors and probability of vertebral body collapse in metastases of the thoracic and lumbar spine. Spine (Phila $\mathrm{Pa}$ 1976). 1997;22(3):239-45.

9. Windhagen HJ, Hipp JA, Silva MJ, Lipson SJ, Hayes WC. Predicting failure of thoracic vertebrae with simulated and actual metastatic defects. Clin Orthop Relat Res. 1997;(344): 313-9.

10. Whyne CM, Hu SS, Lotz JC. Burst fracture in the metastatically involved spine: development, validation, and parametric analysis of a three-dimensional poroelastic finiteelement model. Spine (Phila Pa 1976). 2003;28(7):652-60.

11. Dimar JR 2nd, Voor MJ, Zhang YM, Glassman SD. A human cadaver model for determination of pathologic fracture threshold resulting from tumorous destruction of the vertebral body. Spine (Phila Pa 1976). 1998;23(11):1209-14.

12. Weber MH, Burch S, Buckley J, et al. Instability and impending instability of the thoracolumbar spine in patients with spinal metastases: a systematic review. Int J Oncol. 2011;38(1):5-12.

13. Arana E, Kovacs FM, Royuela A, et al. Spine Instability Neoplastic Score: agreement across different medical and surgical specialties. Spine J. 2016;16(5):591-9.

14. Fisher CG, Schouten R, Versteeg AL, et al. Reliability of the
Spinal Instability Neoplastic Score (SINS) among radiation oncologists: an assessment of instability secondary to spinal metastases. Radiat Oncol. 2014;9:69.

15. Campos M, Urrutia J, Zamora T, et al. The Spine Instability Neoplastic Score: an independent reliability and reproducibility analysis. Spine J. 2014;14(8):1466-9.

16. Fourney DR, Frangou EM, Ryken TC, et al. Spinal instability neoplastic score: an analysis of reliability and validity from the spine oncology study group. J Clin Oncol. 2011;29(22): 3072-7.

17. Leone A, Cianfoni A, Zecchi V, Cortese MC, Rumi N, Colosimo C. Instability and impending instability in patients with vertebral metastatic disease. Skeletal Radiol. 2019; 48(2):195-207.

18. Zadnik PL, Goodwin CR, Karami KJ, et al. Outcomes following surgical intervention for impending and gross instability caused by multiple myeloma in the spinal column. J Neurosurg Spine. 2015;22(3):301-9.

19. Park JH, Kim YB, Hyun SJ, Kang KB, Park PS. Changes in thoracic kyphosis and thoracolumbar kyphosis in asymptomatic Korean male subjects aged $>50$ years: do they progress above T5, T10, T12, or L2? Asian Spine J. 2020;14(2):192-7.

20. Aoude A, Amiot LP. A comparison of the modified Tokuhashi and Tomita scores in determining prognosis for patients afflicted with spinal metastasis. Can J Surg. 2014; 57(3):188-93.

21. Aoude A, Fortin M, Aldebeyan S, et al. The revised Tokuhashi score; analysis of parameters and assessment of its accuracy in determining survival in patients afflicted with spinal metastasis. Eur Spine J. 2018;27(4):835-40.

22. Ulmar B, Reichel H, Catalkaya S, et al. Evaluation and modification of the Tomita score in 217 patients with vertebral metastases. Onkologie. 2007;30(8-9):414-8.

23. Lee $\mathrm{CH}$, Chung $\mathrm{CK}$, Jahng TA, et al. Which one is a valuable surrogate for predicting survival between Tomita and Tokuhashi scores in patients with spinal metastases? A meta-analysis for diagnostic test accuracy and individual participant data analysis. J Neurooncol. 2015;123(2):267-75.

24. Tan T, Donohoe TJ, Huang MS, et al. Does combined anterior-posterior approach improve outcomes compared with posterioronly approach in traumatic thoracolumbar burst fractures?: a systematic review. Asian Spine J. 2020;14(3):388-98. 